Revue des patrimoines

\title{
Le métier de guide-conférencier au service du patrimoine et du tourisme
}

L'identité, la formation et la situation socioprofessionnelle

'Guide-lecturers' in the service of heritage and tourism, identity, training and socio-professional situation

\section{Lina Uzlyte}

\section{OpenEdition}

\section{Journals}

Édition électronique

URL : http://journals.openedition.org/insitu/13654

DOI : $10.4000 /$ insitu. 13654

ISSN : $1630-7305$

\section{Éditeur}

Ministère de la Culture

\section{Référence électronique}

Lina Uzlyte, « Le métier de guide-conférencier au service du patrimoine et du tourisme », In Situ [En ligne], 30 | 2016, mis en ligne le 23 septembre 2016, consulté le 10 décembre 2020. URL : http:// journals.openedition.org/insitu/13654; DOI : https://doi.org/10.4000/insitu.13654

Ce document a été généré automatiquement le 10 décembre 2020.

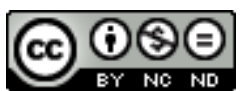

In Situ Revues des patrimoines est mis à disposition selon les termes de la licence Creative Commons Attribution - Pas d'Utilisation Commerciale - Pas de Modification 4.0 International. 


\title{
Le métier de guide-conférencier au service du patrimoine et $d u$ tourisme
}

\author{
L'identité, la formation et la situation socioprofessionnelle \\ 'Guide-lecturers' in the service of heritage and tourism, identity, training and \\ socio-professional situation
}

\section{Lina Uzlyte}

1 Un nouveau statut de guide-conférencier a été instauré par la loi du $1^{\mathrm{er}}$ août 2011 ; nous nous intéresserons à ce qu'il en est en France et tenterons d'analyser les stratégies d'organisation de ce métier dans son environnement social, marqué par le double processus d'une marchandisation croissante de la culture et de sa mise en valeur institutionnalisée. Nous voudrions cerner davantage le statut et le rôle du guide, qui cherche encore son positionnement dans la vaste famille des métiers. Le métier de guide a-t-il une place précise, sa délimitation est-elle nécessaire ? Tend-il à se revivifier ou au contraire, à disparaître ? Comment s'articulent les caractères objectif, subjectif et perçu du métier de guide? Dans quelles conditions ces notions s'opposent-elles ou s'harmonisent-elles?

2 À cet égard, l'étude des politiques du patrimoine constitue un observatoire de premier choix. Le patrimoine est devenu l'interface privilégiée de la culture et de la mémoire. Nous observerons quels enjeux s'y rattachent, tant en termes socio-politiques, économiques et culturels que du point de vue de la transmission d'un savoir qui vise à la construction d'une mémoire individuelle et collective.

3 Le statut de guide-conférencier a obtenu une reconnaissance juridique, dans un premier temps, puis académique. Il évolue au gré des politiques culturelles, elles-mêmes influencées par les pressions exercées par les guides ou divers organismes, et de la transposition du droit communautaire. Un rappel historique s'avère nécessaire à la compréhension des enjeux actuels. 
4 Notre recherche s'appuie sur la réglementation juridique du métier, analyse ses fortes inégalités économiques et cerne le contexte dans lequel il s'inscrit. Afin de montrer ce que revêt ce statut, nous nous sommes aussi intéressée au caractère subjectif de celui-ci.

5 L'idée de cette recherche est venue de quelques interrogations toutes simples : qui est le guide-conférencier, quelle est sa place dans la société ? Quel statut occupe-t-il et quel rôle joue-t-il ? Notre étude permet de mieux comprendre la spécificité de la situation actuelle de guide-conférencier en France.

6 La notion de statut recouvre ici une définition particulière, liée aux droits et aux obligations, aux compétences et aux rôles « joués » autant qu'à l'ensemble des modalités ayant trait au positionnement ou à la place de cette profession dans la société. Le concept de statut renvoie à l'idée d'espace, et plus précisément d'espace social, politique ou économique, tandis que le concept de rôle qui lui est souvent associé implique celle du développement temporel et relationnel d'une suite d'activités procédant de la place occupée dans le milieu social : l'individu exerçant un métier « occupe » un certain statut et il « joue » son rôle.

7 Nous avons pu distinguer le statut objectif, le statut subjectif et le statut accordé ou reconnu. Le statut objectif, traité en premier lieu, est soit juridique, déterminé comme une catégorie juridique des individus selon leur place dans la structure économique, découlant des obligations liées à un certain niveau de revenus ou à l'influence exercée du fait d'un rôle précis dans un domaine de compétence. Le statut subjectif, que nous analyserons ensuite, est une appartenance sociale et se définit par la conception que se fait la personne de sa propre situation dans le système, par rapport aux autres personnes. Le statut accordé, enfin, est la représentation que se font les autres du statut de l'individu.

8 À propos du statut de la profession, nous suivons les idées de C. Dubar et P. Tripier qui considèrent que le terme "profession " possède quatre sens : une déclaration (identité professionnelle), un emploi (classification professionnelle), un métier (spécialisation professionnelle), une fonction (position professionnelle) ${ }^{1}$, quatre sens qui se regroupent sous la notion de situation de la profession. Nous l'utilisons dans son acception la plus large possible pour désigner avant tout la situation ou la place de la profession dans la société.

\section{Le statut objectif}

\section{Cadre juridique}

\section{La création d'un nouveau métier de guide-conférencier}

Une importante réforme des quatre professions réglementées existantes qui assurent la conduite des visites commentées dans les musées et monuments historiques (guideinterprète régional, guide-interprète national, guide-conférencier des Villes et pays d'art et d'histoire, conférencier national) a été engagée avec la parution au JO du décret $n^{\circ}$ 2011-930 du $1^{\text {er }}$ août 2011 relatif aux personnes qualifiées pour la conduite de visites commentées dans les musées et monuments historiques. Ce nouveau décret remplace désormais les quatre professions existantes par une seule, celle de guide-conférencier. Les modalités et conditions d'accès à la profession sont simplifiées. 
10 L'examen national de conférencier national et les examens régionaux sont supprimés au profit d'une formation supérieure diplômante dispensée par des universités ou des établissements d'enseignement supérieur. Une carte professionnelle unique est délivrée aux personnes titulaires d'une certification sanctionnant au moins une formation de niveau licence.

11 Pour exercer son activité, le guide-conférencier doit posséder une bonne culture générale, des connaissances approfondies en histoire, histoire de l'art, ethnographie, économie, géographie. Il a de grandes qualités humaines et relationnelles et le sens de la pédagogie. D'une grande disponibilité, d'une bonne capacité d'adaptation aux attentes des visiteurs, il s'exprime avec aisance dans une ou plusieurs langues étrangères.

12 Le guide-conférencier, synthèse du conférencier et du guide-interprète, conduit des visites, des conférences dans les territoires et les lieux patrimoniaux. C'est un professionnel de la culture et du patrimoine qui assure des visites commentées en français ou dans une langue étrangère.

13 La carte professionnelle est délivrée à chaque personne capable de fournir les attestations demandées dans trois domaines : compétence de guide-conférencier, mise en situation et pratique, langue vivante étrangère.

14 Le métier est également inscrit dans le cadre européen : des aptitudes professionnelles acquises dans un autre État membre de la Communauté européenne ou dans un autre État reconnaissant l'accord sur l'espace économique européen permettent la délivrance d'une carte professionnelle (articles R. 221-15 à R. 221-17 du Code du tourisme²).

\section{Une profession réglementée}

Une lecture attentive de l'article L.221-1, livre II du Code du tourisme, permettrait de dire que le recours à des personnes qualifiées est imposé uniquement dans les musées et les monuments historiques, et ce, de la part des acteurs du tourisme titulaires d'une licence ou habilitées.

\section{Hiérarchisation du métier}

16 Nous tenons à montrer ici comment le métier de guide-conférencier se positionne dans la hiérarchie des métiers en France. Pour voir quel est son poids aujourd'hui, il est nécessaire d'observer son évolution dans le temps en essayant de répondre à la question : le renouvellement contemporain et l'évolution de la réglementation clarifient-elles la situation de cette profession ou au contraire, amènent-elles encore plus de confusion?

Le portail de la fonction publique présente les classements des grades, corps et emplois de l'État. Dans le répertoire des métiers, la profession de guide-conférencier est rangée parmi les services patrimoniaux, au sein de la fonction publique. Son cadre statutaire est déterminé par les catégories $« \mathrm{~B} » \mathrm{ou}$ « $\mathrm{C} \rrbracket^{3}$. Ainsi il se place dans la famille des métiers " établissements et services patrimoniaux 》 (D32), en quatrième position (04/D/32) dans le groupe où figurent directeur d'établissement patrimonial $(01 / \mathrm{D} / 32$, catégorie $\mathrm{A})$, archéologue $(02 / \mathrm{D} / 32$, catégorie $\mathrm{A})$, archiviste, $(03 / \mathrm{D} / 32$, catégorie $\mathrm{A}, \mathrm{B})$, régisseur d'œuvres (05/D/32, catégorie A, B), restaurateur d'œuvres (06/D/32, catégorie A) et agent d'accueil et de surveillance du patrimoine $(07 / D / 32 \text {, catégorie } C)^{4}$. La référence générique qui englobe la profession de guide-conférencier est celle de «médiateur culturel », parmi 
d'autres appellations proposées par ce répertoire comme médiateur du patrimoine, guide de musée, guide-conférencier, animateur du patrimoine, chargé de mission patrimoine.

\section{Formation}

\section{L'accès au métier et sa formalisation par la formation} année, quelque 230 guides-conférenciers sont formés, pour toute la France. Le statut professionnel de guide-conférencier se constitue à travers cette formation qui en trace le "profil» professionnel et le distingue des médiateurs culturels, des animateurs du patrimoine, des conservateurs et des autres métiers. La pluridisciplinarité est une caractéristique de cette formation; les candidats doivent posséder à la fois un solide bagage de culture générale et une expérience professionnelle. L'appellation de guideconférencier recouvre, en France, l'exercice d'une activité professionnelle qui requiert une compétence technique et la détention d'un titre ou d'un diplôme sanctionnant une formation respectant un certain équilibre entre disciplines culturelles, techniques et linguistiques et comportant un minimum d'expérience pratique. La formation dispensée accorde une place importante au patrimoine et aux politiques publiques.

\section{La formation des publics}

La question des enjeux culturels liés à la mise en valeur du patrimoine mérite un traitement particulier dans la mesure où la conception même de patrimoine est liée à la culture. Les politiques du patrimoine sont dépendantes des époques qu'elles traversent. Héritières du siècle des Lumières et de la Révolution française, elles visent à éduquer et instruire le peuple.

Le pédagogue Rachid Amirou défend l'idée selon laquelle la confusion entre loisir et culture s'amorce dès l'école. Par ailleurs, trop souvent, le musée n'est pas considéré par l'enseignant comme un outil au service du savoir, mais plutôt comme une sortie " pédagogique ${ }^{5}$ ». Élisabeth Caillet dit au contraire que c'est parce que le musée n'est pas assez ludique que «si presque tous les enfants vont au moins une fois au musée durant 
leur scolarité, il est amplement démontré qu'à l'âge adulte seuls les individus qui ont suivi des études au-delà du lycée y retournent $»^{6}$. La raison en serait que la visite au musée est assimilée à un cours classique et qu'elle laisse peu de place à l'apprentissage informel, sans évaluation. Cet auteur invoque également les «interdits» qui accompagnent les visites et qui seraient rebutants pour des enfants et des adolescents.

Le développement et la mise en valeur du patrimoine local constituent un atout majeur pour un retour au réel dans la science de la pédagogie. La pédagogie et le tourisme local semblent sur ce point tout à fait conciliables pour penser l'espace vécu, qu'il soit historique ou géographique. Ainsi, grâce au développement et à la mise en valeur du patrimoine culturel local, on peut imaginer que les élèves puissent vivre leur patrimoine et qu'il devienne source de savoir, sans renoncer au plaisir. La visite guidée est une forme de la médiation culturelle qui est construite autour de ce patrimoine. Le guideconférencier est un intermédiaire entre les concepts politiques de la mise en valeur de patrimoine et ses usagers. Il doit donc naturellement surtout connaître et comprendre ce patrimoine pour pouvoir le transmettre.

\section{De l'action culturelle à l'action pédagogique}

La vision globale du métier de guide, d'animateur et de médiateur se reflète dans l'histoire politique culturelle. Le pouvoir et les valeurs de la culture se sont d'abord identifiés aux vertus prêtées à l'éducation. L'éducation se rattache à une volonté politique: former les citoyens. On considère que l'éducation populaire a fait son apparition en 1830, sous le ministère Guizot, au moment où l'État tentait un premier effort d'organisation des classes d'adultes ${ }^{7}$. Elle s'est perpétuée dans la volonté de faire partager les biens culturels. Si l'éducation est un moyen d'adopter et d'assimiler des valeurs sociales, intellectuelles, culturelles et morales, choisies ou subies par l'éducateur et l'institution, on comprend alors combien les effets recherchés et atteints sont liés au modèle de savoir qui les formule. Le modèle du savoir est celui que l'Encyclopédie a mis en place. Cette idée du savoir fonde l'Éducation populaire qui doit contrer la spécialisation et former l'idée de culture véritable "où les diverses sciences sont rigoureusement mesurées et équilibrées, où la science sociale contient et systématise les autres, où domine enfin la Morale, c'est-à-dire la médiation sur le Juste et l'Injuste $»^{8}$. Cette citation résume toute la philosophie de l'éducation jusqu'en 1920. C'est dans ce contexte que se sont instaurés des modes de communication entre ceux qui produisent ou formalisent des idées et ceux à qui elles s'adressent et qui les font avancer.

Avec la loi du 23 avril 1919, dite des "huit heures", se sont ouvertes des perspectives nouvelles pour la diffusion des savoirs et des valeurs à partir d'expériences artistiques. C'est le temps du et pour le loisir lié à la conquête du temps libre. La nouvelle dimension des loisirs est donnée par la politique des loisirs, en 1936, avec le ministère éponyme, dirigé par Léo Lagrange. Ainsi, le petit nombre ou l'élite qui semble disposer de la culture apparait comme l'interprète, le porteur, le dépositaire de valeurs universelles qui meurent lorsqu'elles ne sont pas partagées. Ce principe est développé à travers des supports multiples: bibliothèques, clubs, conférences. Le secteur culturel apparaît comme un moyen d'épanouissement et de libération de l'homme. Dans ce contexte se manifestent les deux métaphysiques de la culture où «Savoir c'est aussi la faculté de Pouvoir ». En même temps, toutes deux participent à l'idée de formation de l'homme, héritée de la Révolution. Il devient alors nécessaire d'éduquer le peuple, de réduire le plus possible les obstacles qui empêchent les hommes d'exercer leur faculté de penser. 
27 La réflexion menée dans ce milieu d'experts s'est concrétisée par la mise en place de services spécialisés dans les diverses administrations publiques et s'est traduite par l'élaboration de politiques culturelles au niveau des États. Dans les années 1990, les nouveaux modes d'intervention, de financement, les législations relatives à l'acquisition, la protection et la conservation du patrimoine culturel ${ }^{9}$ se sont développées. L'émergence de nouveaux métiers a donné lieu à de nombreuses études de spécialistes. Cette évolution a donné lieu à la création de nouvelles formations qui se sont mises en place dans les universités. Les musées semblent (re)devenus le symbole d'une image nationale, régionale ou locale. Ils ont acquis, dans certains cas, un rôle identitaire et sont, dans d'autres, des «lieux de mémoire ${ }^{10} »$.

Le territoire lui-même est amené à se penser du point de vue patrimonial. La découverte du territoire coïncide souvent avec la découverte de soi, de ses origines. Le tourisme culturel à vocation patrimoniale propose à ses visiteurs de s'intéresser à des origines qui ne sont pas les leurs mais grâce auxquelles ils appréhendent un quotidien autre, et peut même leur offrir un enrichissement culturel. Et le guide-conférencier en donne des clés. On peut noter que jusqu'au début $\mathrm{du} \mathrm{xx}^{\mathrm{e}}$ siècle, le tourisme était pratiquement synonyme d'activité culturelle. La culture rejoint désormais le monde marchand en nous amenant vers une autre réalité, avec des défis qui sont désormais d'ordre économique.

\section{Cadre économique}

\section{Panorama économique}

29 Pour de nombreuses collectivités, le patrimoine touristique figure parmi les éléments majeurs de leur attractivité, source d'activités et de développements économiques, facteur de cohésion de leurs identités locales, support de créativité et d'innovation. C'est pourquoi chaque collectivité souhaite, en France comme dans les pays qui accueillent un tourisme de masse, constituer un modèle à part entière en matière de valorisation et de médiation du patrimoine dans un but de « mise en tourisme $»^{11}$.

30 Les métiers du patrimoine mettent en exergue les savoir-faire français et ils contribuent fortement à l'attractivité touristique des territoires. Ceux qui analysent le tourisme culturel du point de vue économique s'intéressent au tourisme culturel en termes de marchés, et donc à l'offre de tourisme culturel et ses composantes, son marketing et son développement.

31 L'itinéraire est une pierre angulaire du tourisme culturel et reflète à la fois sa conception et son organisation : le guide-conférencier joue un rôle crucial dans la difficile association entre tourisme et culture, entre authenticité et efficacité commerciale ${ }^{12}$. Ici le guideconférencier est une composante $\mathrm{du}$ "produit touristique ", ensemble de prestations associant transport, hébergement, restauration, encadrement, animation, assurance et assistance.

Les opérateurs publics et privés, nationaux et internationaux ont élaboré de nouveaux moyens, doctrinaux (chartes et conventions), juridiques (inventaire, classement) et techniques (mesures de protection, gestion des flux, mise en valeur, promotion, renforcement de la participation des populations locales) permettant d'assurer une gestion plus équilibrée. À cet effet, des "plans de gestion » sont élaborés qui incluent l'ensemble des mesures de protection et de mise en valeur. Et l'analyse des attentes et des comportements des visiteurs passe par une observation du terrain, mise en place par la 
direction des musées de France, le musée du Louvre, le Centre des monuments nationaux, la Cité des sciences et autres centres d'accueil du public qui produisent périodiquement des données descriptives: origine géographique, statut socioprofessionnel, âge, statut familial, sensibilité à la communication, fidélisation, tarifs, satisfaction, suggestions. Sur le plan financier, les retombées économiques des visites guidées sont intégrées dans les recettes annexes de l'établissement. Ces recettes représentent une partie importante des produits d'exploitation, entre $20 \%$ et $50 \%$ du chiffre d'affaires dans les monuments, musées et sites historiques visités ${ }^{13}$.

Selon la structure où le guide travaille, il peut être libéral, salarié, auto-entrepreneur ou vacataire. Comme les guides sont libres d'effectuer autant de visites guidées qu'ils le peuvent, ils possèdent souvent deux voire trois statuts fiscaux, ce qui leur assure un certain niveau de revenus.

Les obligations découlant du statut juridique dominant dans la profession telle qu'elle est réglementée dépendent beaucoup du type d'employeur choisi par les professionnels. Les guides-conférenciers sont souvent sollicités pour des prestations de courte durée par des employeurs ou des donneurs d'ordre très variés. La durée moyenne d'une prestation, d'après l'étude de cabinet Richard Lewy Consultant, est de 3 heures ${ }^{14}$. D'après cette étude, seuls les deux tiers des personnes qui ont répondu à cette enquête exercent cette activité à titre unique chez un ou plusieurs employeurs. Lorsqu'elles exercent le métier de guide à titre principal, leurs activités complémentaires relèvent de l'accompagnement, de la fonction d'accueil, de l'enseignement ou du développement touristique ou culturel.

L'activité de visite guidée revêt un caractère saisonnier; cette saisonnalité est plus marquée dans le secteur touristique, puisque les flux sont liés aux vacances ou périodes de fêtes, variables d'un pays à l'autre, mais s'étalant sur 5 à 6 mois. Les employeurs et les guides-conférenciers répondent à des demandes ponctuelles. Cette combinaison de saisonnalité et de ponctualité produit un effet naturel de périodes de demandes plus ou moins abondantes dites basse et haute saisons. Ainsi, la vacation reste le statut dominant pour cet emploi. Les guides déclarent en moyenne 549 heures de travail pour cette activité.

Ce secteur est très dépendant des conjonctures économiques et politiques nationales et internationales. Crise économique ou conflit diplomatique ont des répercussions immédiates sur l'offre des agences de voyages et les tour-opérateurs. Les turbulences qui affectent les environnements nationaux et internationaux sont devenues telles qu'il n'existe plus de situation ou de position dominante relativement durable; les avantages concurrentiels restent précaires, particulièrement pour les industries proposant des services de nature touristique.

Les recommandations de l'étude sur laquelle nous nous sommes appuyés ouvrent pour nous une problématique générale : comment en est-on arrivé à une telle situation? Par intérêt des uns, ou manque de vigilance des autres? Faudrait-il laisser la profession assumer ses libertés ou au contraire, la contraindre ? Est-il nécessaire de la protéger et de la consolider davantage?

\section{L'institution culturelle et le tourisme}

Le secteur dans lequel le guide-conférencier travaille rythme son activité, et finalement se reflète sur son salaire. 
39 Le domaine de l'économie de la culture a pris en compte la valorisation touristique mais nous pouvons toutefois noter que le tourisme culturel a été longtemps absent dans l'élaboration de la politique culturelle en France qui se concentre plutôt sur le public national. Le plus grand nombre d'informations recensées sur le tourisme provient justement du milieu institutionnel et des professions du tourisme qui, étant liées à une industrie marchande, ont développé très tôt et bien avant les institutions culturelles une culture du chiffre et des stratégies qui s'y rattachent.

À première vue, il semble que ce sujet soit peu abordé et que le musée à sa naissance était un projet patrimonial, du moins tel qu'il fut conçu lors de la création des monuments nationaux et des premiers musées. Il est pensé d'abord comme lieu de la transmission des pères aux enfants, où le patrimoine est une succession dans la continuité du temps entre le passé et l'avenir ${ }^{15}$. En France, le peuple est destinataire de cet héritage. Cette histoire se joue sur plusieurs siècles et nous pouvons observer là l'évolution d'une conscience touristique. En 1966, date des premières enquêtes sociologiques et de l'ouvrage de Bourdieu $^{16}$, il est devenu clair que la fréquentation de ces lieux patrimoniaux se répartissait entre les visites des groupes scolaires et les amateurs vieillissants. Le public étranger était amené par les tour-opérateurs qui les prenaient en charge. C'est ainsi que la promotion de la pratique de visite du patrimoine a été en grande partie organisée par les professionnels du tourisme. Peu à peu, les agences de voyages sont devenues les détentrices du choix des itinéraires. Elles sont devenues également les prescripteurs de l'organisation des visites guidées pour finalement prendre une place dominante, via leurs guides-interprètes multilingues, dans l'interprétation même des monuments et des collections. On peut remarquer qu'à l'époque, les groupes n'étaient pas bien vus, les touristes non plus. Ils étaient considérés comme « un mal nécessaire » et qu'il s'agissait d'un public qui n'était pas formé à l'offre des institutions culturelles en Europe.

\section{Le tourisme culturel}

41 Nous allons d'abord examiner comment s'est manifesté à travers le temps le processus qui a mis en relation les hommes et leur espace culturel, définissant ce que l'on appelle aujourd'hui « tourisme culturel ». La relation établie entre « tourisme » et " patrimoine » est récente mais on peut faire remonter le concept de voyage culturel à l'Antiquité.

Nous pouvons dire que cette tendance s'est accentuée de nos jours, car d'après l'OCDE, " plus de $50 \%$ de l'activité touristique en Europe est générée par le patrimoine culturel et le tourisme culturel devrait être la composante du secteur du tourisme à connaître la plus forte croissance ${ }^{17}$. Mais la définition du tourisme culturel n'est pas évidente. On peut en trouver toute une déclinaison, avec des variantes selon les années, les sources et les rédacteurs ${ }^{18}$; nous avons donc choisi celles qui contribuent surtout à préciser la place du guide-conférencier dans ce monde. Les définitions proposées par le site d'Atout France (Agence de développement touristique de la France) rendent compte de cette réalité contrastée et des enjeux de l'action conjointe entre acteurs du tourisme et de la culture. Par extension, on y inclut les autres formes de tourisme où interviennent des séquences culturelles. Le tourisme culturel est donc une pratique culturelle qui comprend un déplacement d'au moins une nuitée, ou que le déplacement va favoriser. Les chercheurs du programme européen PICTURE élargissent encore la notion du tourisme culturel, ajoutant que

c'est aussi une forme de tourisme centré sur la culture, l'environnement culturel

(incluant les paysages de la destination), les valeurs et les styles de vie, le 
patrimoine local, les arts plastiques et ceux du spectacle, les industries, les traditions et les ressources de loisirs de la communauté d'accueil. Il peut comprendre la participation à des événements culturels, des visites de musées et monuments et la rencontre avec des locaux. Il ne doit pas seulement être considéré comme une activité économique identifiable, mais plutôt comme englobant toutes les expériences vécues par les visiteurs d'une destination au-delà de leur univers de vie habituel ; cette visite doit durer au moins une nuitée et moins d'un an, se passer dans un hébergement privatif ou marchand de la destination ${ }^{19}$.

Les guides-conférenciers interviennent ici comme accompagnateurs des visiteurs, médiateurs entre les visiteurs et ce monde à découvrir et ambassadeurs des pays dont ils vont faire découvrir le patrimoine.

4 L'estimation des retombées économiques est souvent fondée sur les dépenses des touristes. Pour mesurer l'efficacité des investissements publics ou privés au niveau national, il faut tenir compte des facteurs socio-économiques : le patrimoine historique est aussi un facteur non négligeable de ressources directes et indirectes, d'emplois et donc de développement des territoires et participe à la hausse du PIB. D'une certaine façon, le tourisme culturel est l'avenir du tourisme et donc indirectement, l'avenir du métier de guide-conférencier ${ }^{20}$. Les liens entre l'économie et la culture cherchent à se renforcer dans le monde entier. Ainsi, en Europe, la Commission européenne promeut le tourisme culturel. Elle le fait parfois afin de conforter "l'unité dans la diversité » de la population européenne. Voyager pour découvrir la culture de l'autre permet d'apprécier les différences culturelles.

\section{Le statut subjectif}

\section{Le statut social et les rôles}

5 Certains sociologues comme Mendras ou Circourel font appel à la notion de statut global pour mettre en lumière l'articulation qui préside à l'ensemble des statuts et des rôles qu'assume l'individu. En effet, un individu tient plusieurs rôles étant donné que la société lui confère plusieurs statuts. Le statut est l'aspect normatif (constitué de droits et de devoirs) activé par le rôle car c'est lui qui enclenche le processus d'actions et de comportements caractéristiques.

Maisonneuve montre que le rôle a une "fonction de régulation des rapports sociaux » puisqu'il correspond à un état du système social organisé en statuts, ajuste les relations sociales par le « jeu » que permettent les rôles assignés aux individus, identifie les conflits (de rôles), indices d'autres conflits de statut (entre guide et médiateur ou conservateur, entre guide et public)...

Le rôle transforme l'action individuelle en action sociale parce que l'exercice d'un rôle suppose l'exercice corrélatif d'autres rôles. Par exemple, le rôle de guide a besoin du rôle du public à qui il s'adresse. Dans le processus de formation de rôle, l'institutionnalisation est importante puisqu'elle détermine les droits, les devoirs et les obligations qui découlent du rôle. D'après Jacqueline Nadel, il permet d'accéder au statut; il relève de l'image ou du stéréotype que les gens ont en tête à propos du statut. Le rôle se définit donc en termes d'attentes et d'actions par rapport au statut.

La recherche effectuée par Aurélie Peyrin sur le statut social des médiateurs lui permet d'affirmer que « le fait de décrire ce que l'on fait, faute de pouvoir dire qui l'on est ${ }^{21}$ » est 
juste en ce qui concerne le nom des médiateurs. Dans le cas des guides-conférenciers, les réponses des guides à une demande de se présenter et décrire leur métier sont nuancées. Le nom de «guide » s'est imposé au fil du temps et ainsi tous les guides interrogés ont répondu qu'ils étaient "guides, tout simplement", mais souvent en précisant qu'ils étaient guides-étudiants en histoire de l'art, guide-médiateur, guide-ancien professeur ou encore guide-comédien. Parfois, il nous semble que dire « je suis guide » ne suffisait pas, comme s'il était préférable d'avoir une activité autre qui justifie le fait d'être guide. Souvent, comme le dit le guide-conférencier Danièle, les touristes demandent aux guides « quel est votre « vrai » métier? »...

49 Nous avons présenté les réponses qui selon nous correspondent à un « état » du métier, et à travers lesquelles nous constatons que la situation de la France est particulière, puisque le guide appartient autant au monde du tourisme qu'à celui de la culture, qu'il prend luimême des initiatives pour sauvegarder son apport au patrimoine culturel commun et en encourager le développement et que la défense de ses droits reste une démarche personnelle : «La spécificité du métier, c'est la multiplicité des collections et des musées. Ce qui veut dire beaucoup de travail personnel en dehors face public ».

50 C'est un métier très spécifique avec au minimum deux caractéristiques. Le guide est à la fois un spécialiste de la pédagogie et de l'information.

51 Voici quel profil se dessine à travers les interviews avec les guides, les concepteurs des visites-guidées, et les responsables des structures.

Comment reconnait-on un conférencier?

53 - «par son amour pour la parole, son besoin d'être face à un public et par sa grande timidité »,

54 - « c'est un métier et une passion et ce n'est pas toujours évident de dissocier les deux. Ce qui compte, c'est la volonté de faire partager ce plaisir à regarder ",

55 - « c'est vraiment une amplitude de thèmes et de type de public que nous touchons qui est exceptionnelle »,

- « nous sommes recrutés pour notre savoir scientifique d'abord, après humain ».

57 Dans son livre Une vie au pays des touristes, Danièle Louveau-Jouan, riche d'une expérience de plus de quarante ans comme guide-interprète, nous présente un portrait sur le vif du guide en France. Elle nous dit :

Certains de nos clients cultivés ont honte du mot « guide» et voudraient nous présenter sous leurs programmes sans y accoler ce fâcheux substantif. Ils nous demandent d'être historiens de l'art, ou de la culture, voire conférencière des Monuments historiques. Mais non, chers clients amis, il n'y a pas d'autre nom à notre métier. Je suis guide-interprète nationale. Je l'assume. Et j'en suis même très fière ${ }^{22}$.

Nous voyons ainsi que ce que veut dire guide pour un juriste ou une institution culturelle n'est pas forcément la même chose pour un visiteur et est encore autre chose pour un guide.

La vision la plus large du guide est donnée par Michèle Gellereau dans son ouvrage Les mises en scène de la visite guidée $e^{23}$ :

Chacun de nous a en tête les images caricaturales du guide bavard, incompréhensible qui, sous une avalanche de dates et d'anecdotes vous interdit de toucher aux tapisseries. Mais chacun de nous se souvient aussi d'un guide extraordinaire, qui dans un discours passionné vous a fait comprendre l'essence 
d'un lieu ou d'une œuvre, a changé votre regard sur les choses. On pourrait alors les désigner comme les «parents pauvres » des médiations patrimoniales et muséales, tant la fragilité de leurs statuts et la diversité des conditions d'exercice est peu « en phase » avec le rôle réel qu'ils jouent sur le plan patrimonial.

\section{Position sociale du guide-conférencier}

60 Nous interrogeant sur le statut social du guide, nous allons nous poser les questions de sa place sur l'échelle socioprofessionnelle. Quels sont les rapports avec son environnement et de quelle façon agissent-ils sur son statut? Qui est le guide pendant la visite-guidée, quel est son rôle? Comment se positionne-t-il, pendant ce temps donné à la découverte, par rapport au lieu et à son public? Où est sa place dans les logiques publiques? Comment le guide se comporte-t-il pour réussir à ne pas créer de conflits et les résoudre s'ils se produisent?

61 D'après Aurélie Peyrin, la médiation dans les musées recouvre un ensemble de services d'accompagnement des visiteurs qui prennent la forme de conférences, visites guidées, ateliers... Nous ajoutons que l'accompagnement des touristes hors des musées est la même médiation sauf qu'il recouvre les services d'accompagnement privés, de tourisme. Ces services peuvent s'appuyer sur des formes d'interactions différenciées selon le public visé. Les acteurs de cette médiation sont des animateurs, guides-conférenciers ainsi que les professionnels qui conçoivent et organisent les activités. Le rôle social de la médiation est de donner des clés d'interprétation dans des domaines variés; la société professionnelle dans laquelle se trouve le guide-conférencier est donc constituée de tous ces acteurs. Et la question qui nous intéresse au sein de cette organisation porte sur comment leurs relations agissent sur le statut de guide-conférencier et donc sur son comportement et les attitudes professionnelles.

62 Il est important de dire que le statut social de guide dépend de celui à partir duquel il transmet l'information, de celui à qui il la communique et dans quel but. Comme la plupart sont vacataires, les guides-conférenciers constituent un groupe peu homogène. Le guide est caractérisé par sa position d'intermédiaire entre un visiteur et un lieu.

Les guides interrogés ont eu des difficultés à déterminer le rôle que joue le guide dans leur société professionnelle, ou même, pire, les guides-interprètes répétaient qu'ils n'avaient ni rôle ni statut reconnu ou du moins, qu'il était peu respecté. Il semble donc que la profession de guide-conférencier n'a pas de place fixe et confirmée dans l'échelle sociale. Il existe un écart entre la réglementation qui définit le statut des guidesconférenciers, a priori claire, et la position réelle de la profession, si mal connue et reconnue dans la société contemporaine. Les guides expriment le sentiment que c'est une profession bien réglementée mais peu considérée.

Revenons à Michèle Gellereau, dont l'étude apporte une vision du statut de guide dans le domaine de la communication et de la médiation. Selon elle, le guide définit son rôle et fait reconnaître sa place dans le jeu des interactions. Dans la situation de visite, le concept de "place » proposé par F. Flahaut et cité par M. Gellereau est intéressant pour notre compréhension puisqu'il permet d'étudier comment des positions statuaires externes à l'interaction et des positionnements internes construisent un jeu de places négociables qui influence la relation entre ceux qui interagissent : chacun accède à son identité à partir et à l'intérieur d'un système de places qui le dépasse ; ce concept implique qu'il n'est pas de parole qui ne soit émise d'une place et n'assigne une place à l'interlocuteur, 
soit que cette parole présuppose uniquement que le rapport de places est en vigueur, soit que le locuteur en attende la reconnaissance de sa place propre ou oblige son interlocuteur à s'inscrire dans ce rapport ${ }^{24}$. L'attitude que le guide a envers le visiteur est donc construite à l'intérieur d'un ensemble où le statut communicationnel permet d'occuper une place qui se négocie constamment ${ }^{25}$.

\section{L'impact des publics sur le statut de guide}

Pour les différents publics auxquels le guide s'adresse, il reste une personne étroitement liée au lieu qu'il représente. Il sera toujours celui qui le connaît le mieux. C'est au tour du guide de se poser à chaque fois le problème, contraignant, de son statut, du type de relation qu'il s'autorise, de sa position, de son rôle d'intermédiaire entre le lieu où il travaille et les visiteurs dont la réceptivité est aléatoire et tributaire de facteurs divers et variés (géographiques, climatiques, intérieurs et extérieurs, liés à leur âge, ou à la situation physique, etc.). Autant de questions auxquelles le guide doit faire face, avec sa situation et ses difficultés propres.

\section{Le statut à la carte}

Des lieux différents vont attirer des publics différents. Dans un espace organisé, le guide a moins de latitude et sa position est déterminée par le lieu d'accueil, souvent institutionnalisé, où la visite doit s'inscrire dans une logique globale. À partir du moment où le guide doit respecter un texte, ou une trame imposée, il représente l'institution et prend en charge un discours qu'il aura tendance à défendre face au visiteur. Mais en extérieur, dans la visite d'une ville par exemple, dans le respect de l'ordre public, le guide est libre d'incarner plusieurs statuts selon le message qu'il veut faire passer à ses interlocuteurs. Bien sûr, le plus simple serait de se référer à son statut juridique de guideconférencier, mais souvent les guides se trouvent face à des personnes qui sollicitent un guide pour accéder à la vie "authentique», quelqu'un de local qui fait partie de l'aventure du voyage. Ainsi le guide va-t-il se présenter comme la personne liée à l'endroit, comme un habitant ou un témoin appartenant à un lieu précis dont il est en quelque sorte l'âme. Parce que sa position, par rapport à ce qu'il présente, ne relève pas seulement d'un statut professionnel mais aussi de ses liens à l'environnement. Une guideconférencière nous a dit que « la visite-guidée doit être comme un conte, qui permet de se détacher des a priori, et nous ouvrir. Ainsi, même des années après, les visiteurs vont se rappeler des paysages qu'ils ont vus. Nous agissons sur la conscience de nos touristes ». Ou encore, Marie, guide, qui dit que «l'alchimie de la visite se crée en écoutant aussi la demande du groupe, pour mieux y répondre. Nous, les guides, nous n'avalons pas des quantités de connaissances. Le but ce n'est pas d'avoir, c'est d'être devant le public ».

Ces avis soulignent que l'attitude de guide se construit dans l'interaction avec et par rapport à son auditoire. Ce lien est spécifique en son effet du « filtre » personnel. Michèle Gellereau nous le confirme, parlant de négociation permanente des positions, et elle évoque le phénomène de " poser les oreilles » ou « être un écran » que provoque le guide, ce qui ensuite sera traduit par les effets de cette forme de la médiation plus tard.

Nous empruntons encore à $\mathrm{M}$. Gellereau son illustration du besoin d'intimité du visiteur qu'elle tire du film de Roberto Rossellini Voyage en Italie (1954) :

L'héroïne a présélectionné ce qu'elle veut voir, elle veut se protéger de l'inconnu mais cherche aussi à garantir l'intégrité de son imaginaire (souvenirs douloureux et 
problèmes conjugaux) : elle n'arrive pas à coller ce qu'elle voit et ce qu'elle entend, le discours rassurant des guides. À un moment le guide ne veut pas l'amener où elle veut aller (voir le temple d'Apollon), elle s'échappe et, seule, ne voit que des images qui la renvoient à ses problèmes. comme une contrainte, mais aussi comment il peut obliger le visiteur à se décentrer de ses propres préoccupations et s'ouvrir au monde à découvrir.

\section{La logique du public}

Les études et les stratégies muséales ont fait évoluer la notion de public. Celui-ci est segmenté, fragmenté et sectorisé : les scolaires, les jeunes, les retraités, les chômeurs, les groupes, les touristes nationaux ou internationaux, le public appartenant à différents secteurs professionnels, les visiteurs individuels ou en famille, les handicapés, les amateurs, les professionnels des musées, les journalistes, les enseignants, les étudiants, les amis des musées, les adhérents, les représentants des diverses communautés ${ }^{26}$.

71 Pour certains spécialistes, la relation des individus à la culture - qu'il s'agisse d'art ou de science - est très différenciée et cela n'est pas suffisamment pris en considération par les musées $^{27}$, tout comme la distinction entre visiteurs nationaux et étrangers qui reste marquante. Tout cela entraîne deux logiques d'action et crée une politique du public à deux vitesses. Ainsi, les objectifs du guide peuvent être de satisfaire un public/clientèle qui profite des agréments d'un lieu ou bien d'avoir une démarche médiatrice qui privilégierait l'éducation d'un public/citoyen à qui il faut donner les clefs d'accès au savoir, à la mémoire.

72 Les programmes de séjours sont organisés dans le cadre des multiples accords entre les professionnels du tourisme et les acteurs de l'infrastructure accueillant les visiteurs ce qui donne lieu à des programmes très chargés qui prennent rarement en compte le voyageur. La guide Sophie, qui a commencé sa carrière dans une agence qui organisait l'accueil des touristes ajoute que «le futur guide devrait absolument faire le stage dans les deux postes, dont l'un est celui de tour-opérateur et l'autre, de l'autre côté ». Certes, le guide rencontre des situations encore plus différenciées sur le terrain : il est amené à travailler dans un car ou un bateau, à utiliser un microphone, ce qui modifie un peu le contact. L'interaction se fait en équipe avec un autre professionnel du tourisme comme le guide-accompagnateur ou le chauffeur du bus. Et puis l'architecture de la visite ainsi que son rythme vont être pensés comme une dynamique entre l'information et l'expérience, entre le savoir et le plaisir.

73 Ce contexte, marqué par une grande diversité des situations, incite à s'interroger sur le rôle spécifique que jouent les guides pour faire vivre les lieux culturels et patrimoniaux et sur leur place dans un dispositif plus global d'accompagnement des visiteurs. On peut appréhender cette question sous l'angle de l'ingénierie culturelle ou encore celui de la pédagogie, quand il s'agit de choisir des modes de médiation visant à améliorer l'accompagnement des visiteurs.

\section{Les rôles des guides}

La rencontre se déroule dans un cadre culturel, social, politique, un environnement physique - lieu, temporalité - qui ne permet pas aux acteurs de jouer n'importe quelle partition. Un guide évolue dans un centre d'interprétation très organisé. C'est la fonction 
des statuts, qui anticipent sur les comportements et servent aussi d'évaluation : être guide-conférencier, ou un habitant de la ville, ou le propriétaire du lieu, par exemple, influence non seulement la place que le guide prend dans la visite mais aussi les idées qu'il développe. La diversité et la multiplication des structures dans lesquelles se déroulent les visites guidées jouent autant sur la relation qui va s'instaurer avec les visiteurs que sur le contenu du discours. Les guides vont se servir des lieux pour réaliser une mission ou développer une stratégie d'influence.

Revenons vers la définition de rôle et considérons cette fois celle d'E. Goffman : « on peut appeler rôle ou routine le modèle d'action préétabli que l'on développe durant une représentation et que l'on peut présenter et utiliser en d'autres occasions ». Analysant la manière dont les individus engagés dans une interaction jouent le rôle exigé par la situation dans laquelle ils évoluent, il définit «le rôle social comme l'actualisation de droits et de devoirs attachés à un ou plusieurs rôles ${ }^{28} »$.

Si nous ajoutons la liberté d'expression, par ces critiques nous pouvons déduire que les rôles à jouer peuvent s'envisager sous l'angle des tâches à exécuter pour interpréter les lieux mais aussi sous l'angle des conceptions que l'employeur ou les visiteurs ont de ce qui est attendu du guide lors de la visite. Michèle Gellereau se réfère, pour la notion d'interprétation, à D. Jacobi et A. Meunier et dit que l'interprétation peut travailler dans trois dimensions: "expliquer, traduire, créer. L'interprétation de rôle comme explication, au sens de trier, démêler, dévoiler, révéler correspond à la posture du spécialiste, de l'expert, du savant ou à la rigueur du pédagogue ; au sens traduire à la neutralité et à l'efficacité de la communication et créer à jouer sur l'émotion et la sensibilité ${ }^{29}$. Les rôles choisis vont susciter des comportements différents.

Lors des entretiens avec les guides, les professeurs qui les forment et les professionnels qui les encadrent, nous avons découvert que ce n'est pas seulement le montant du salaire qui garantit la qualité de la visite-guidée. La motivation est ici aussi un critère très important: cette activité ne se fait pas seulement pour gagner de l'argent ou pour s'occuper l'esprit. Il ne convient pas non plus d'endosser le rôle de la personnalité effacée ou celle du bon confesseur. Il ne suffit pas de préférer la paix à la guerre, ni de se positionner comme un maître de jeu ou un médecin des âmes. Le point de vue du guideconférencier existe. Il est clair et affirmé, fondé sur une éthique ${ }^{30}$. D'après les compétences exigées et enseignées aux guides-conférenciers, la classification et la hiérarchisation du métier et les autres aspects analysés, nous pouvons déduire que le métier de guide peut être vu comme la spécialisation de différents types de médiation. Il serait donc intéressant de voir les liens qu'il entretient avec cette discipline, fondée sur les compétences du médiateur lui-même.

\section{En quoi le guide est un médiateur}

Le guide se doit de ne pas départager, juger ou conseiller mais d'établir une communication d'échange. Le guidage, comme la médiation, se développe dans de multiples contextes ${ }^{31}$. On peut élargir la notion de contexte au sens que lui donne Quéré : « En un sens, le contexte est le tout ou le champ en fonction duquel une action, un geste, une parole, un événement ou un objet acquièrent une intelligibilité, un sens, une individualité $»^{32}$. lors évidente. Ces professionnels sont des «généralistes» de la circulation de 
l'information entre des personnes. Face à l'interprétation des faits, ils permettent la clarification ; face au rejet et au prêt d'intention, ils favorisent tous les deux l'émergence de la qualité ; enfin, face à l'esprit de contrainte, ils créent le contexte de la créativité, qu'il s'agisse des relations culturelles et politiques, professionnelles, économiques, ou sociales. Dans la transmission des connaissances le guide peut se positionner en médiateur entre le « découvrant » et le sujet qu'il propose de découvrir. Cette pratique professionnelle de la médiation, dans les relations interpersonnelles directes, nécessite de la part du professionnel une maîtrise de savoir-faire et de savoir-être qui ne peut s'acquérir que dans le cadre d'une formation.

80 Les interventions des guides peuvent être de différentes natures. Les guides ne vont pas seulement informer des visiteurs mais créent aussi des interrelations. Cette action est efficace parce qu'elle s'en tient aux techniques spécifiques, en premier lieu, au métier, et finalement à la médiation. D'après les témoignages multiples de professionnels engagés dans ce métier, il parait clair que la reconnaissance et l'acceptation de la légitimité des points de vue et des positions sont les clés de la réussite.

Le statut de guide fait qu'il se retrouve toujours à l'épicentre des différents conflits entre la culture cultivée et culture de masse, dans la création comme rupture, d'après Jean Caune $^{33}$, entre le monde de la culture et le monde commerçant, jusqu'aux micro-conflits intérieurs entre un ton strict ou des plaisanteries, dans le positionnement envers ses collègues, etc. où les parties en conflit souvent ne se connaissent pas ou connaissent très peu les limites strictes de leur action.

Les conflits esthétiques sont chargés d'une violence qui n'est pas seulement symbolique ; ils mettent en jeu des positions affectives et intellectuelles ainsi que des postures sociales vis-à-vis des œuvres commentées. Les œuvres provoquent aussi bien fusion que séparation, connivence que rejet. Le guide doit tenir compte du fait que le jugement de goût, la critique qui peut être exprimée, visant des objets qui s'adressent à la sensibilité, déterminent des activités de loisir, filtrent les perceptions des choses de la vie et par conséquent, construisent nos choix affectifs.

Ici la neutralité est une qualité professionnelle. Cette neutralité doit cependant être active et sans ambiguïté et dépend de l'expérience personnelle et de la volonté de rester créatif, philosophe et de faire preuve de tact face aux autres. Dans la dynamique conflictuelle, la confiance en soi est vitale. Le public est très attentif au comportement du guide et un climat particulier s'établit souvent dès les premières minutes d'intervention.

Pour illustrer ces propos, nous tenons à citer ceux de Sophie, guide qui travaille majoritairement avec le public américain des croisières :

Souvent, dans mes groupes, il y a des historiens ou autres spécialistes des thèmes abordés tout comme des personnes qui ne connaissent pas grand chose sur ces sujets. Il est très important de répondre aux attentes des connaisseurs sans dévaloriser la méconnaissance des autres. C'est un vrai défi pour un guide ! Parfois j'encourage "ceux qui savent » à intervenir dans mes commentaires en tant que spécialistes tout en donnant un rôle d'observateurs ou d'amateurs aux autres. Il ne faut surtout pas se laisser embarquer dans le jeu de la compétition « qui sait plus ». 
son acceptation n'est pas une soumission à l'autorité qu'il pourrait représenter aux yeux de l'une ou l'autre des parties en conflit.

Dans tous ces moments, le guide accomplit le rôle de médiateur. Toutefois, lors de la visite ou de la conférence, le guide-conférencier devrait maîtriser ces techniques et être dans l'observation très fine des "foyers à risque ». Lors de sa visite, le guide crée un contexte d'écoute dont le processus valorise le respect des autres.

La visite guidée réussie est une forme de médiation $^{34}$ qui est tournée vers l'enrichissement culturel des personnes à travers des émotions, les ressentis de leur place dans l'histoire, à travers le contact humain, la rencontre et le sentiment de partage, le vivre-ensemble. Pour cela il faut d'abord veiller à ce que s'instaure un climat qui favorise la confiance. Il ne s'agit pas d'animer mais de permettre des échanges en restant centré sur chacun, d'où la nécessité de recourir de façon maîtrisée à des techniques relationnelles, notamment celle de l'altéro-centrage ${ }^{35}$, sans privilégier une approche qui serait psychologique, juridique, thérapeutique ou encore sociologique. La médiation « ouvre les portes».

89 Comme les bustes de Mercure, le dieu des intermédiaires, qui étaient placés au croisement des routes et à l'entrée des villes, l'activité de guide-conférencier se situe au point de rencontre des connaissances (sciences sociales), de l'enseignement (pédagogie), de la créativité (mise en scène de sa visite guidée, relations interpersonnelles, résolution de problème), de l'accompagnement.

En dix ans, la médiation, héritière des recherches sur les causes des conflits et leur résolution ${ }^{36}$, a pris un réel essor. Aujourd'hui issue de pratiques très diverses, elle constitue un ensemble cohérent, créant le métier de " faiseurs de paix », les médiateurs ${ }^{37}$. Et sur ces chemins de la médiation, nous rencontrons les guides, les psychologues, les sociologues, les enseignants, les avocats, les notaires, les huissiers et de nombreux consultants et formateurs car c'est une pratique très ancienne qui concerne une multitude d'activités et touche à tous les domaines que les professions citées ne représentent pas dans leur totalité. Le guide peut s'éloigner du médiateur culturel, mais il se reconnait surtout dans une vision de la médiation qui établit des liens en les créant, les renouant et les rétablissant. Nous pourrons d'ailleurs constater que le concept de médiation tisse un lien entre la qualité de la communication et de l'ensemble de savoirfaire et savoir-être qui lui sont attachés. Il est donc légitime de retrouver ce concept relié au social, au politique, à l'économique comme à l'enseignement et aux services culturels.

91 L'accompagnement des visiteurs en tant que forme de médiation ainsi que la médiation elle-même sont partout mais ils ne sont pas formalisés, ce qui les met face aux crises et aux rapports de force.

Par ailleurs, quand les interactions sont réussies, le guide supporte facilement un emploi du temps qui peut sembler très lourd. La position des guides en tant que professionnels n'est pas toujours claire et la précarité de leur statut administratif et juridique les contrarie, provoquant des débats douloureux parce qu'elle est ressentie comme une forme d'indifférence à l'égard de ceux qui défendent ce système ou même le représentent aux yeux des autres.

93 Nous souhaitons reprendre les idées de Pierre Besnard ${ }^{38}$ à propos de l'éducation tout au long de la vie : le doute systématique, nécessaire à l'esprit d'ouverture et de recherche propre au métier de guide, lui évite de se nourrir des théories stériles, bureaucratiques, ou idéologiques, et le préserve de l'esprit de système, d'où vient son charme. Enfermer ce 
métier dans les cadres trop stricts aboutirait à l'étouffer. Cependant, le reconnaître en lui accordant une place digne parmi les autres métiers du patrimoine et le préserver face aux défis globaux, politiques ou économiques serait très important.

Cette activité, qui s'affirme de plus en plus comme une nouvelle pratique sociale interactionnelle incontestable, a ses institutions, ses publics, ses agents, ses promoteurs et concepteurs. À partir des données analysées, nous constatons que la réforme des statuts de guide était un premier pas sur la voie d'évolution de ce métier et son insertion dans l'espace social.

Nous voudrions rappeler ici l'aphorisme d'Alfred Korzybski « une carte n'est pas le territoire ${ }^{39} »$ qui signifie qu'une carte ne sera jamais aussi précise et complète que le territoire qu'elle représente, que la loi n'est pas le métier. Le territoire évolue en permanence et saisir ses changements serait impossible. Il y aura toujours des éléments manquants. Ce fait nous amène à réfléchir au lien entre nous-mêmes et notre propre perception du monde par rapport à la réalité, qui n'est elle-même rien d'autre qu'une représentation intérieure de la perception extérieure du monde. Pourtant, la coopération culturelle, essentielle à la cohésion de l'Europe, est aujourd'hui une nécessité absolue.

\section{NOTES}

1. - DUBAR, Claude, TRIPIER, Pierre. Sociologie des professions. Paris: Armand Colin, coll. «Collection U », 1998, p. 251.

2. - Les définitions de la DGCIS (direction générale de la Compétitivité, de l'Industrie et des Services), voir le site: http://archives.entreprises.gouv.fr/2012/www.tourisme.gouv.fr/ profession/metiers/guidage.html [consulté le 14/09/2016].

3. - Catégorie « $\mathrm{B} »$ correspond au niveau bac ou bac +2 et emploi comportant des fonctions d'application (par exemple : IDE, DEASS, BTS ou DUT...) ; catégorie « $\mathrm{C} »$ : sans diplôme ou niveau infra bac (BEPC, CAP, BEP) et emploi comportant des fonctions dites d'exécution. Voir le site : http://www.emploitheque.org/plan.php [consulté le 19/08/2016].

4. - Voir fiche métier, disponible en ligne sur le site du CNFPT : http://www.cnfpt.fr/node/146/ repertoire-metiers/sous-famille/36?mots_cles=04/D/32\&gl=ODUwMGE2Y2I [consulté le 19/08/2016].

5. - AMIROU, Rachid. Imaginaire du tourisme culturel. Paris : PUF, 2000, p. 35.

6. - CAILLET, Élisabeth. À l'approche du musée, la médiation culturelle. Lyon : PUL, 1995, p. 127.

7. - CACÉRÈS, Benigno. Histoire de l'Éducation populaire. Paris : Le Seuil, 1964, p. 46.

8. - Ibid., p. 68.

9. - GIRARD, Augustin. Développement culturel : expériences et politiques. Paris : Unesco, 1977.

10. - NORA, Pierre (dir.). Les lieux de mémoire. t. I, La République [1984]. Paris : Gallimard, 1993, " Entre Mémoire et Histoire », p. 18.

11. - CAZES, Georges, LANQUAR, Robert, RAYNOUARD, Yves. L'aménagement touristique. [1980]. Paris : PUF, 1993, p. 14.

12. - BORET, Alain. «Itinéraires de tourisme ». Dans ORIGET DU CLUZEAU, Claude. Le tourisme culturel. Op. cit., p. 60.

13. - PATIN, Valéry. Tourisme et patrimoine [1997]. Paris : la Documentation française, 2005, p. 153. 
14. - LEWY, Richard. Étude sur l'exercice des métiers de guide-interprète et conférencier. Ministère de l'Économie, Avignon, 2009, p. 29. Voir le site: http://archives.entreprises.gouv.fr/2012/ www.tourisme.gouv.fr/stat_etudes/etudes/entreprises/etude_guide_interprete.pdf [consulté le 19/08/2016].

15. - BALLÉ, Catherine, POULOT, Dominique. Musées en Europe, une mutation inachevée. Paris : la Documentation française, 2004, p. 13.

16. - BOURDIEU, Pierre, DARBEL, Alain. L'amour de l'art : les musées et leur public. Paris : les Éditions de Minuit, coll. «Le sens commun », 1966.

17. - La Voix du Patrimoine Culturel en Europe, voir le site : http://www.europanostra.org/whowe-are/ [consulté le 19/08/2016].

18. - Atout France, définition du tourisme culturel, voir le site : http://www.pch.gc.ca/pc-ch/ pubs/tourism/documents/2006-05/4_f.cfm [consulté le 19/08/2016].

19. - Le groupe de recherche de l'UAM, le glossaire du projet ICOMOS, ICTC, 2002, disponible sur Internet : http://www.picture-project.com/glossaire_list.php3?id_rubrique=17 [consulté le 19/08/2016].

20. - Voir le site : http://www.canalu.tv/video/universite_de_tous_les_savoirs/ le_tourisme_culturel_claude_origet_du_cluzeau.1468 [consulté le 19/08/2016].

21. - PEYRIN, Aurélie. Être médiateur au musée: sociologie d'un métier en trompe-l'œil. Paris: la Documentation française, 2010, p. 75.

22. - LOUVEAU-JOUAN, Danièle. Une vie au pays des touristes. Questembert: Stéphane Batigne éditeur, 2012, p. 10.

23. - GELLEREAU, Michèle. Les mises en scène de la visite guidée : communication et médiation. Paris/ Budapest/Turin: L'Harmattan, 2005, Introduction. PEYRIN, Aurélie. «Les paradoxes de la médiation culturelle dans les musées ». Informations sociales, 2012/2 $\mathrm{n}^{\circ} 170$, p. 62-65.

24. - FLAHAUT, François. La parole intermédiaire. Paris : Le Seuil, 1978, p. 58.

25. - GELLEREAU, M. Les mises en scène des visites guidées. Op. cit., p. 70.

26. - DI MAGGIO, Paul. "Social Structure, Institutions, and Cultural Goods: the Case of the United States". Dans BOURDIEU, Pierre \& COLEMAN, James. Social Theory for a Changing Society. Boulder: Westview Press, 1991.

27. - GOTTESDIENER, Hana. Évaluer l'exposition: définitions, méthodes et bibliographie commentée d'études d'évaluation. Paris : la Documentation française, 1987, p. 31.

28. - GOFFMAN, Erving. La mise en scène de la vie quotidienne. t. II, Les relations en public [1972]. Paris : Éditions de Minuit, 1973, p. 23.

29. - JACOBI, Daniel. Dans GELLEREAU, M. Op. cit., p. 91.

30. - Ethics \& Social Dimension of Tourism [Le Code mondial d'éthique du tourisme], voir le site : http://ethics.unwto.org/fr/content/le-code-mondial-d-ethique-du-tourisme [consulté en août 2013].

31. - CHAUMIER, S., MAIRESSE, F. La médiation culturelle. Paris : Armand Colin, 2013, p. 61.

32. - QUÉRÉ, Louis. «La situation toujours négligée ? ». La coopération dans les situations de travail. Réseaux, vol.15, $\mathrm{n}^{\circ} 85,1997$, p. 184, voir le site: http://www.persee.fr/doc/ reso_0751-7971_1997_num_15_85_3139 [consulté le 19/08/2016].

33. - CAUNE, Jean. La démocratisation culturelle : une médiation à bout de souffle. Grenoble : Presses universitaires de Grenoble, 2006, p. 78.

34. - CHAUMIER, S., MAIRESSE, F. La médiation culturelle. Paris : Armand Colin, 2013, p. 115.

35. - L'altéro-centrage est une application de l'énoncé de René Descartes : « Ne pas prendre pour soi ce qui ne l'est pas » qui consiste dans l'aide à la fixation de l'attention, du soutien à la réflexion et à la prise de décision. Il s'agit d'une posture éthique et déontologique, ce qui en fait plus une méthode qu'une technique. Le néologisme a été créé par Jean-Louis Lascoux en 2000. Il a commencé à être utilisé en dehors des formations de médiateurs professionnels à partir de 2005. 
Quoiqu'il soit utilisé de diverses manières, il a son sens plein dans le contexte des relations professionnelles d'entretien ou d'animation de réunion.

36. - BOUTHOUL, Gaston. Essais de polémologie [1976]. Paris : Denoël/Gonthier, coll. « Bibliothèque Médiations ", 2000, p. 60.

37. - LASCOUX, Jean-Louis. Pratique de la médiation : une méthode alternative à la résolution des conflits . Issy-les-Moulineaux : ESF éd., 2001, p. 16.

38. - BESNARD, Pierre. L'animation socioculturelle. Paris : PUF, 1980.

39. - KORZYBSKI, Alfred. Une carte n'est pas le territoire : prolégomènes aux systèmes non-aristotéliciens et à la sémantique générale [1998]. Paris : Éditions de l'Éclat, 2007, p. 46.

\section{RÉSUMÉS}

L'activité de guide-conférencier est actuellement en pleine évolution: il nous a semblé intéressant d'analyser la position et l'identité socio-professionnelle de cette profession dont les compétences sont mises au service du patrimoine. Nous avons voulu voir quelle était son évolution et observer comment ce métier participait à la mise en valeur du patrimoine culturel. À travers l'accueil et l'accompagnement des publics, les guides-conférenciers offrent une réponse à la transformation de la culture et de son environnement. Ils reflètent le caractère du territoire dont ils sont les représentants et donnent des clefs à ses visiteurs pour le comprendre. Ils s'intègrent ainsi à la notion plus vaste de médiation culturelle. C'est dans ce contexte que se situe notre étude, qui analyse l'interaction entre le visité, le visiteur et l'interprète considérée du point de vue du guide conférencier - médiateur culturel.

Rapid change in the profession of guides can be observed today. It seemed interesting to understand the identity and position of this profession, its socio-professional composition, and to observe its evolution in the cultural heritage sector. This study will offer some interpretation of the social, cultural and political context of tourist guiding and how it is organised in France. Guides react to the evolution of culture and its environment, accompanying their audience. They translate the mentality of the territory they belong to and represent and they make it accessible to visitors. They fit into the broader concept of cultural mediation which is also the context of our study. It analyses the interaction between the visited, the visitor and the interpreter from the tourist guide-cultural mediator point of view.

\section{INDEX}

Keywords : profession, tourist guide, status, cultural mediation

Mots-clés : métier, guide conférencier, statut, médiation culturelle

\section{AUTEUR}

\section{LINA UZLYTE}

Doctorante en communication et information, CERLIS-UMR 8070, Sorbonne Nouvelle - Paris 3 maruzli@yahoo.fr 